\title{
Cervical Spondylotic Myelopathy due to the Ochronotic Arthropathy of the Cervical Spine
}

\author{
Nan Li, M.D., Wei Tian, M.D., Ph.D., Qiang Yuan, M.D., Da He, M.D. \\ Department of Spine Surgery, Beijing Jishuitan Hospital, Beijing, China
}

Ochronosis is a musculoskeletal manifestation of alkaptonuria, a rare hereditary metabolic disorder occurs due to the absence of homogentisic acid oxidase and leading to various systemic abnormalities related to deposition of homogentisic acid pigmentation (ochronotic pigmentation). The present case reports the clinical features, radiographic findings, treatments and results of a cervical spondylotic myelopathy woman patient due to the ochronotic arthropathy of the cervical spine. The patient aged 62 years was presented with gait disturbance and hand clumsiness. Physical examination, X-rays, computed tomography and lab results of the urine sample confirmed the presence of ochronosis with the involvement of the cervical spine. The patient underwent a modified cervical laminoplasty due to multi-segment spinal cord compression. The postoperative follow-up showed a good functional outcome with patient satisfaction. The present study concludes the conditions and important diagnostic and surgical aspects of a patient. It is necessary to identify the condition clinically and if cord compression is observed, appropriate surgical interventions needs to be instituted.

Key Words : Ochronosis · Alkaptonuria · Cervical spine.

\section{INTRODUCTION}

Alkaptonuria (AKU) is a rare hereditary autosomal recessive disease characterized by a defect in the gene which codes for homogentisate-1, 2 dioxygenase resulting in the metabolic disorder of the aromatic amino acids, phenylalanine and tyrosine ${ }^{7)}$. One in a million is born with these birth defects. The enzymehomogentisic acid oxidase catalyzes the conversion of homogentisic acid (HGA) to maleylacetoacetic acid, and its absence leads to increase in HGA accumulation in the body. Some of the excess HGA excretes through the urine which turns dark on exposure to oxygen or alkalization occurs. Rest of accumulated HGA oxidizes initially, and deposits within the connective tissue irreversibly and subsequently turns into melanin-like pigment. It occurs especially in the cartilage of the joints and in the intervertebral discs, which is named as ochronosis ${ }^{11,14}$.

Ochronosis not only affects the cardiovascular, genitourinary and respiratory systems, but also results orthopedic disorders like spondylosis and arthropathy. The disease progresses from simple alkaptonuria to alkaptonuric ochronosis, leading to ochronotic arthropathy ${ }^{10}$. Cervical myelopathy is usually due to degenerative changes in the spine with the osteophytes from the poste- rior part of the vertebral body and uncinate process encroaching upon the neural canal. Numerous reports on the successful management of ochronotic arthropathy of the peripheral joints like the knee and hip joints using total joint replacement. However, there is a paucity of reports that have shown successful surgical intervention for spinal especially cervical spine involvement.

In this paper, we report a woman patient suffering from ochronotic spondylosis who was diagnosed during the late course of disease once the spondylitic myelopathy had set in resulting in increased neck and back pain, and associated with walking trouble and hands clumsiness.

\section{CASE REPORT}

A 62-year-old woman was admitted in the hospital in June 2011, with a diagnosis of cervical spondylotic myelopathy. The patient began to experience neck and back pain with stiffness 7 years ago. The symptoms developed slowly, until the gait disturbance and hand clumsiness began to appear in 2010. Initially she received conservative treatment and it was less effective. However, her symptoms progressed and at the time of presentation she was wheel chair bound. She didn't have any history of trauma

- Received : August 27, 2014 • Revised : January 3, 2015 •Accepted : January 5, 2015

- Address for reprints : Wei Tian, M.D., Ph.D.

Department of Spine Surgery, Beijing Jishuitan Hospital, 31 Xinjiekou Dongjie, Beijing 100035, China

Tel : +86-1058516934, Fax : +86-1058516934, E-mail : spinejs@@126.com

- This is an Open Access article distributed under the terms of the Creative Commons Attribution Non-Commercial License (http://creativecommons.org/licenses/by-nc/3.0) which permits unrestricted non-commercial use, distribution, and reproduction in any medium, provided the original work is properly cited. 
with no relevant past medical or similar family history.

Physical examination showed cyan pigmentation on the nose, sclera, and the ear auricle and also in other parts of the body (Fig. 1). There was kyphosis at the thoracolumbar segment, resulting in a restricted movement at neck and back. Positive Romberg and Tandem gait sign and the finger-escape sign were observed. The grip-and-release test was 8 movements at both hands in 10 seconds, which was obviously inferior to the normal criterion of above 20 movements. Paresthesia on both hands and radial side of the left forearm and no motor weakness was observed. No pathological reflexes were observed on either side. The preoperative Japanese Orthopedic Association myelopathy (JOA) score for her was 7.

CT scans indicated multilevel degenerative disc disease from cervical to lumbar spine, including narrowing, calcification, osteophytosis and vacuum phenomenon (Fig. 2, 3). MRI showed the cervical spinal compression in multi-segment by disc extrusion and ligamentum flavum redundancy simultaneously (Fig. 4).

As the color of the patient's urine turn to dark after exposure to air after two hours, the patient's urine sample was screened for metabolic disorder. The measured value for HGA was 1220.03 while the normal value should be 0 . So, we confirmed the presence of alkaptonuria.

As the patients' cervical spine had normal lordosis and multilevel stenosis, we performed the modified laminoplasty. This

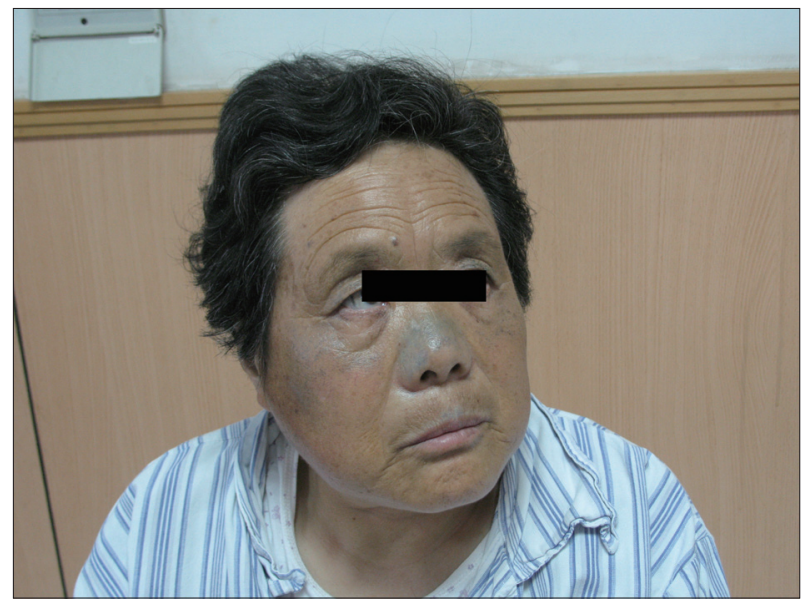

Fig. 1. Typical ochronotic pigmentation secondary to deposition on the nose, sclera and jowl.
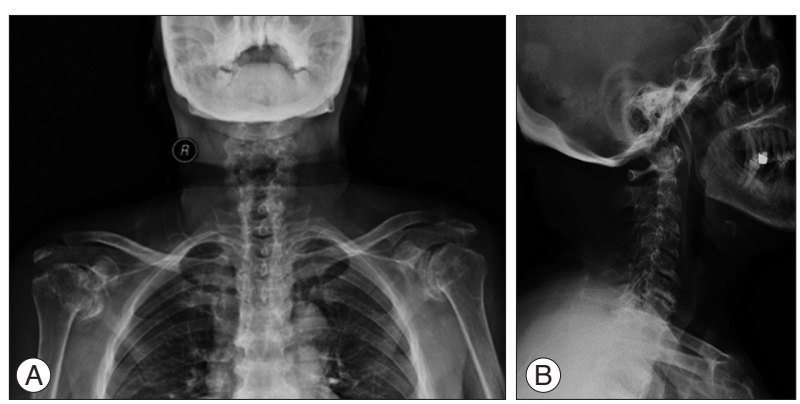

Fig. 2. The AP (A) and lateral (B) view of the cervial spine showing reduced disc spaces, pronounced disc calcifications and spondylosis. involves C3 laminectomy, C7 upper semi-laminotomy, and C4-6 open-door laminoplasty with implantation of man-made coral bone interposition. This was in contrast to the conventional cervical laminoplasty technique that involves a laminoplasty from C3-7 completely. During the operation, the ossificated nuchal ligament and the ligamentum flavum between $\mathrm{C} 2-3$ were stained black in color due to the deposition of the oxidized HGA pigmentation (Fig. 5). After surgery, the patient recovered uneventfully (Fig. 6). The cervical JOA score improved from 7
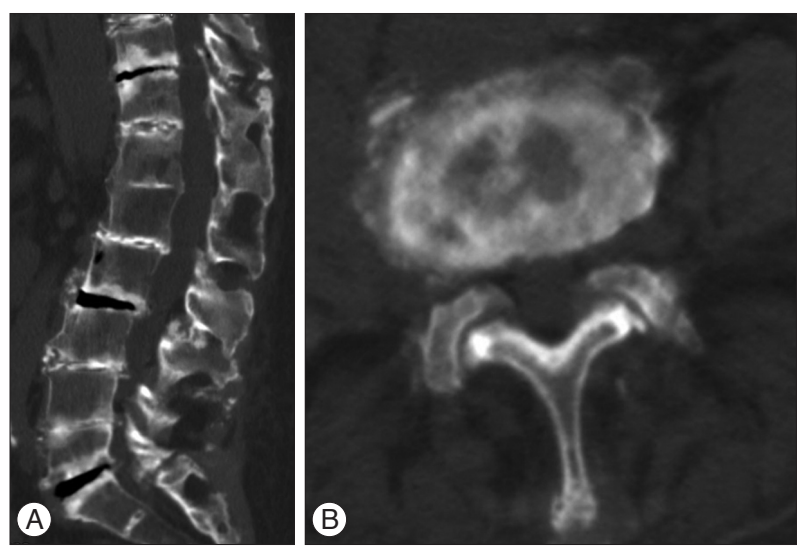

Fig. 3. Sagittal (A) and axial CT (B) view of the lumbar spine showing the typical vacuum phenomenon and profound water-like calcification with sparing of the central nucleus pulposus.
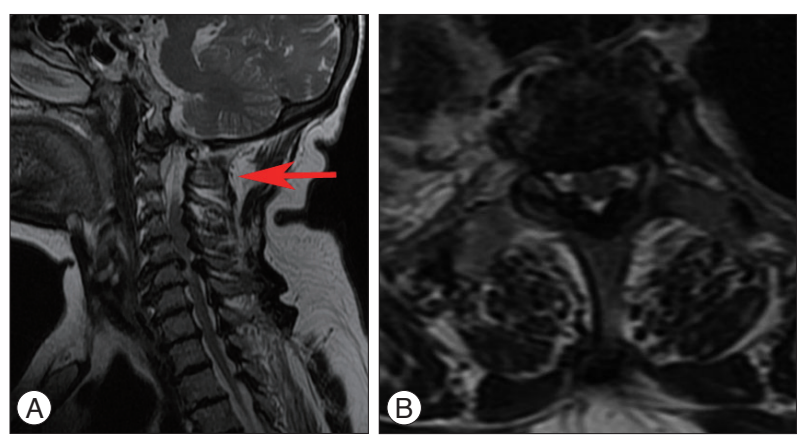

Fig. 4. A and $B$ : The cervical MRI show the spinal cord is compressed at multi-level mainly from the redundant ligamentum flavum posteriorly (C2 : arrow head).

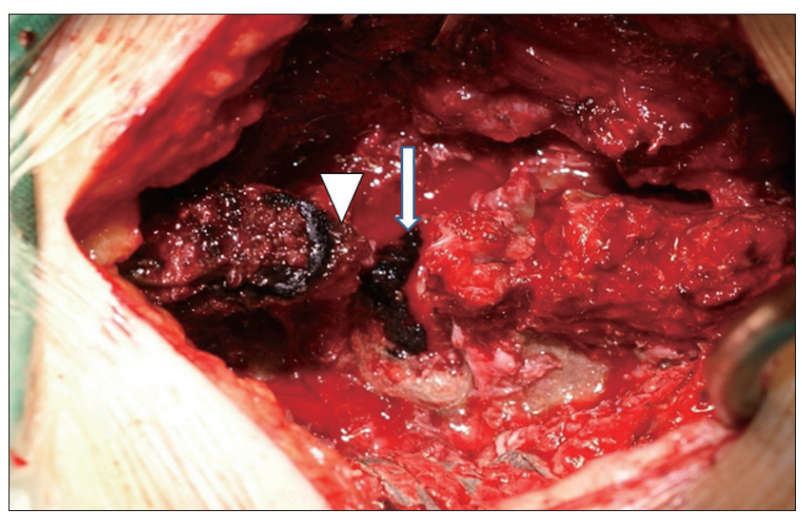

Fig. 5. Black oxidized HGA pigmentation (long arrow) is found in the ligamentum flavum between the C2 (arrowhead)-3 lamina. 


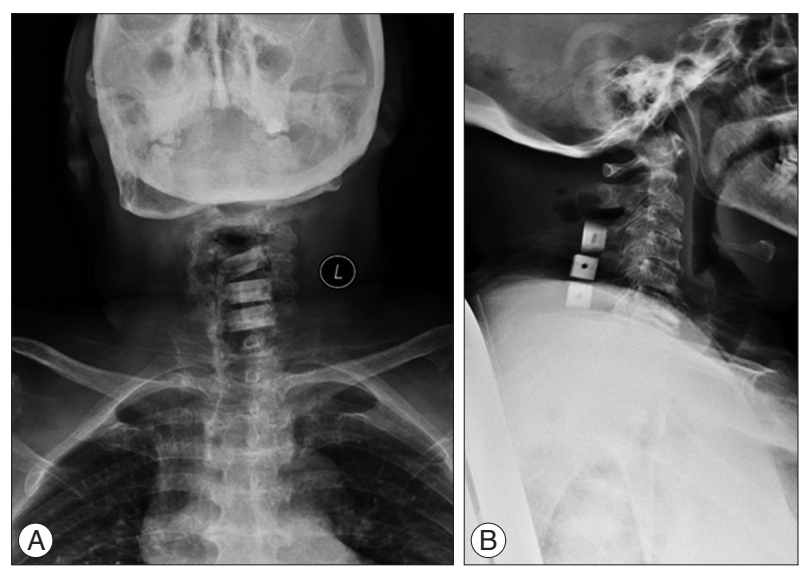

Fig. 6. $A$ and $B$ : The $X$-ray picture of the laminoplasty procedure postoperatively, which shows the artificial wedged coral bone interposition from the $\mathrm{C} 4$ to the $\mathrm{C} 6$.

preoperatively to 9 at discharge and reached 11 at one year postoperatively. She was satisfied with the treatment although she had difficulty in climbing stair case without crutches.

\section{DISCUSSION}

The cervical spondylotic myelopathy (CSM) is not an uncommon condition in the spinal area, which follows a protracted clinical course consisting of a longer period of relatively stable symptoms punctuated by exacerbation of variable duration. For patients with moderate to severe symptoms, the surgical intervention should be considered as a possible procedure to prevent further neurologic deterioration and create a chance for recovery from myelopathy ${ }^{4,5,12)}$.

$\mathrm{AKU}$, with its sequel ochronosis is a rare disease caused by an inborn deficiency of HGA oxidase. Although there are symptoms that may give instant clue to the diagnosis like the urine turns to dark on exposure to air and discoloration on sclera, jowl, as well as in the underwear, the patients can often neglect the symptoms and appear at late in the course of disease $\mathrm{e}^{2,4)}$. In few cases it may be neglected until fourth or fifth decade when the symptoms secondary to ochronosis develop and the patients report to physicians. The disease affecting the spine follows a chronological sequence, the patient usually first suffer from ochronotic spondylosis which is accompanied with the peripheral joint arthropathy ${ }^{1)}$, and it progresses to cervical spondylotic myelopathy which warrants surgical intervention. The initial complaints usually include low back or neck pain with stiffness, and occasionally sciatica resulting from disc protrusion ${ }^{6}$. Involvement of large joint usually occurs several years after the spinal disorders ${ }^{10)}$.

The characteristics of ochronotic spondylosis are multiple disc space narrowing, vacuum phenomenon and calcification in a water-like pattern due to the sparing of the central nucleus pulpous ${ }^{3,9)}$. Current literature has rarely reported the involvement of the cervical spine and consequent spinal cord compression requiring surgical intervention. In the present case report, findings from the MRI on the cervical region revealed that the redundant ligamentum flavum appeared to be the main cause behind the cord compression. This could be secondary to the fact that the HGA could bind irreversibly by a process of polymerization and oxidation to connective tissue collagen. This prevents the collagen cross-link formation, which leads to the abnormality of the ligamentum flavum ${ }^{3,8,14}$.

In view of the findings from MRI, we modified the standard technique to perform a modified cervical laminoplasty, which can permit extensive decompression through posterior expansion of the canal while continually maintaining cervical alignment and stability through the preservation of the posterior elements. There is no specific treatment for alkaptonuria. Nowadays, all the available methods are only symptomatic for the earlystage, such as dietary restriction of tyrosine and more intake of vitamin $\mathrm{C}$ by its antioxidant effect, etc ${ }^{3,13)}$. Although the ochronotic arthropathy can severely handicap the patient because of its involvement in the spine and other joints, this incidence is quite rare. Earlier surgical intervention is needed when the ochronosis results in spinal cord dysfunction. Cervical laminoplasty is often the procedure of choice given the fact that the involvement is extensively causing multilevel compression.

\section{CONCLUSION}

$\mathrm{AKU}$ is a rare metabolic genetic disorder that is caused by the deficiency of homogentisic acid oxidase, affecting multiple organ systems in the body. A typical cervical spondylotic myelopathy due to the ochronotic arthropathy of the cervical spine was reported in this paper, and a modified laminoplasty proved to be the appropriate treatment that yielded satisfactory result.

\section{References}

1. Alrehaily A, Pope JE : Alkaptonuria with atypical joint involvement. J Rheumatol 29: 198-199, 2002

2. Aynaci O, Onder C, Turhan AU : Bilateral hip arthroplasty for ochronotic arthropathy. Clin Rheumatol 19: 150-152, 2000

3. Chua SY, Chang HC : Bilateral spontaneous rupture of the quadriceps tendon as an initial presentation of alkaptonuria--a case report. Knee 13: 408-410, 2006

4. Corrà T, Zaccala M, Galante $\mathrm{M}$ : Ochronotic arthropathy : rapid destructive hip osteoarthritis associated with metabolic disease. Clin Rheumatol $14: 474-477,1995$

5. Dom K, Pittevils T : Ochronotic arthropathy : the black hip. Case report and review of the literature. Acta Orthop Belg $63: 122-125,1997$

6. Emel E, Karagöz F, Aydín IH, Hacísalihoğlu S, Seyithanoğlu MH : Alkaptonuria with lumbar disc herniation : a report of two cases. Spine (Phila Pa 1976) 25 : 2141-2144, 2000

7. Fernández-Cañón JM, Granadino B, Beltrán-Valero de Bernabé D, Renedo M, Fernández-Ruiz E, Peñalva MA, et al. : The molecular basis of alkaptonuria. Nat Genet 14 : 19-24, 1996

8. Gaines JJ Jr : The pathology of alkaptonuric ochronosis. Hum Pathol 20 : 40-46, 1989

9. Justesen P, Anderson PE Jr : Radiologic manifestations in alcaptonuria. Skeletal Radiol 11 : 204-208, 1984 
10. Kusakabe N, Tsuzuki N, Sonada M : Compression of the cervical cord due to alcaptonuric arthropathy of the atlanto-axial joint. A case report. J Bone Joint Surg Am 77 : 274-277, 1995

11. Van Offel JF, De Clerck LS, Francx LM, De Jonge MJ, Stevens WJ : A patient with ochronotic arthropathy and spondylopathy : a difficult differential diagnosis with spondylitis ankylosans. Clin Exp Rheumatol 13 : 259261, 1995

12. Van Offel JF, De Clerck LS, Francx LM, Stevens WJ : The clinical mani- festations of ochronosis : a review. Acta Clin Belg $50: 358-362,1995$

13. Wolff JA, Barshop B, Nyhan WL, Leslie J, Seegmiller JE, Gruber H, et al. : Effects of ascorbic acid in alkaptonuria : alterations in benzoquinone acetic acid and an ontogenic effect in infancy. Pediatr Res 26 : 140-144, 1989

14. Zhao BH, Chen BC, Shao de C, Zhang Q : Osteoarthritis? Ochronotic arthritis! A case study and review of the literature. Knee Surg Sports Traumatol Arthrosc 17 : 778-781, 2009 